\title{
Efeito agudo da estimulação vibratória em hemiparéticos espásticos pós-acidente vascular encefálico
}

\author{
Janaína de Moraes Silva, Mario Oliveira Lima, Alderico Rodrigues de Paula Júnior
}

Resumo Acidente Vascular Encefálico (AVE) é o termo usado para caracterizar uma perturbação focal da função cerebral, de suposta origem vascular isquêmica ou hemorrágica, que tem como principal manifestação clínica a espasticidade. O estímulo vibracional constitui um novo recurso terapêutico para a reabilitação, visando à modulação dessa disfunção tônica. Objetivou-se investigar os efeitos imediatos e de curta duração da estimulação vibracional na espasticidade e atividade muscular, do membro inferior de sujeitos hemiparéticos. Foram selecionados para compor a amostra 27 sujeitos, de ambos os sexos. Estes foram submetidos à goniometria e eletromiografia, antes e depois da estimulação vibratória aplicada por intermédio de uma almofada digital. Atribuiu-se para os testes estatísticos, o nível de significância de 5\%. Os resultados sugerem que houve um aumento significativo, da amplitude de movimento $(\mathrm{p}<0,0001)$. A atividade eletromiográfica apresentou um aumento significativo entre o valor de pré-vibração e pós cinco minutos do término da vibração no músculo gastrocnêmio $(p<0,05)$ e tibial anterior $(p<0,01)$ e pós dez minutos do término da vibração em ambos os músculos $(\mathrm{p}<0,01)$. Houve um aumento, porém não significativo do RMS quando comparado à fase prévibração e pós-vibração imediata. Pode-se concluir que a estimulação vibratória interferiu na modulação do tônus, na atividade muscular, promovendo o ganho de amplitude de movimento da articulação do tornozelo dos sujeitos avaliados.

Palavras-chave Acidente vascular encefálico, Espasticidade muscular, Vibração.

\section{Acute effect of vibratory stimulation in spastic hemiparetic after a stroke}

\begin{abstract}
The stroke (CVA) is the term used to characterize a focal disturbance of cerebral function, of supposed ischemic vascular or hemorrhagic origin, which has spasticity as the main clinical manifestation. Vibrational stimulus is a new therapeutic agent for rehabilitation, which aims to change this tonic dysfunction. This research aims at investigating the immediate and short-term effects of vibrational stimulation on spasticity and muscular activity of hemiparetic subjects'lower limb. A sample of 27 subjects of both sexes with post stroke symptoms was selected. They were subjected to goniometry and electromyography, before and after vibratory stimulation applied through a digital pad. The 5\% significance level was attributed to the statistical tests. The results suggest that there was quite a significant increase in the range of motion $(p<0.0001)$. The electromyographic activity increased significantly between the pre-vibration value and five minutes after the end of vibration in the gastrocnemius muscle $(p<0.05)$ and tibialis anterior $(p<0.01)$ and ten minutes after the end of vibration in both muscles $(p<0.01)$. There was an increase, though not significant, in Root Mean Square (RMS), when compared to the immediate pre-vibration and post-vibration. One may conclude that the vibratory stimulation interfered with tone modulation in muscle activity, promoting gain in the evaluated subjects' ankle joint range of motion.
\end{abstract}

Keywords Stroke, Muscle spasticity, Vibration. 


\section{Extended Abstract}

\section{Introduction}

Stroke (CVA) is the term used to characterize a focal disturbance in the brain function of supposed ischemic vascular or hemorrhagic origin. The stroke clinical manifestations are related to the location and extension of vascular injury. Corticospinal changes promote a deficiency in motor control interfering in daily life activities, as a result of spasticity and muscle weakness. These corticospinal changes decrease the patient's ability to produce and regulate volunteer movement. Studies suggest beneficial effects of somatosensory stimulation to minimize interferences like spasticity and muscle weakness in subjects with central nervous system injury. Vibration has multiple physiological influences, which promote normal patterns of motor activity caused by the excitability of motoneuron modulations. The vibrations increase the afferent final flow of the primary muscle spindle, allowing tonic vibration reflex. Recently, vibration producing devices have been developed and studies have proved their efficiency. This study aims at investigating the immediate and short-term effects of vibrational stimulation in spasticity and muscle activity in hemiplegic subjects'lower limb after stroke. The article justifies itself not only because of the basic science production but also because of technological resources applied from many areas such as engineering.

\section{Material and Methods}

The study was carried out with 27 male and female subjects with stroke diagnosis. They were submitted to goniometry and electromyography before and after vibrational stimulation with a vibratory digital pad (commercially available equipment). The vibration was continuously applied for 15 minutes and the electromyographic collections were realized before the vibration, immediately after, 5 minutes after, and 10 minutes after the end of the vibration.

\section{Results}

The 27 participating patients were between 46-75 years old $(64.4 \pm 4.24)$ with average post stroke time of $33.7 \pm 12.00$ (12-60 months). They included 25 men and two women, and as for the type of stroke, 17 had ischemic etiology and ten had hemorrhagic stroke. As for the affected hemibody, 17 had with right-sided hemiparesis and ten had left-sided hemiparesis.

The results suggest that there was a significant increase in the ankle dorsiflexion range of motion in the affected hemibody $(p<0.0001)$. The electromyographic activity using the Root Mean Square (RMS) parameter presented a significant increase between the pre-vibration value and five minutes after the vibration in the gastrocnemius muscle $(p<0.05)$ and tibialis anterior $(p<0.01)$ and ten minutes after the vibration in both muscles $(p<0.01)$. There was an increase, however not significant, of RMS when compared with the pre-vibration phase and immediately after vibration.

\section{Discussion}

The skeletal muscle is a tissue specialized in modifying its functional capacity in response to different stimuli. The human body replies to vibration in a complex way because it imposes a hypergravital activity in relation to the high acceleration. The mechanical action of the vibration produces fast and short changes in the length of the complex tendinous muscle. Thus, the three motor effects are the result of the vibration stimulus in the muscle. The first is a sustained contraction known as a tonic reflex to the vibration, in which the muscle in the vibration process actively contract, as a result of stimulating the muscle spindles. Second, reciprocal inhibition depresses the motoneural excitability innervating the antagonist muscles. Third, the monosynaptic tract of the myotatic reflex in vibration musculature is repressed.

Exposure to the vibration causes temporary changes in the excitability of central and peripheral neural structures, probably facilitating the activation of motor units immediately after the stimulus application and improving motor performance.

\section{Conclusions}

In tune with the experimental protocol used, vibration has shown to increase the degree of ankle dorsiflexion range of motion, the electromyographic activity for the tibialis anterior and medial gastrocnemius muscles after the application of vibratory stimulation. Further research is necessary to understand how vibration stimuli act on physical parameters. 


\section{Introdução}

O Acidente Vascular Encefálico (AVE) é a terceira causa de morte em diversos países. Suas vítimas, se não forem a óbito, poderão apresentar sequelas físico-funcionais significativas (Bear et al., 2002).

As manifestações clínicas do AVE estão relacionadas a localização e extensão da lesão vascular. As lesões no sistema corticoespinal promovem déficit no controle motor interferindo nas atividades de vida diária. (O`Sullivan e Schmitz, 2010), em decorrência da espasticidade e fraqueza muscular. Há uma diminuição da habilidade do paciente em produzir e regular o movimento voluntário (Corrêa et al., 2005), principalmente durante a deambulação, merecendo destaque ao déficit na realização do movimento de dorsiflexão do tornozelo durante a marcha, devido à fraqueza do músculo tibial anterior e a espasticidade do músculo tríceps sural (Soares, 2003).

A espasticidade pode ser definida como aumento velocidade-dependente do tônus muscular com exacerbação de reflexos profundos, decorrente da hiperexcitabilidade do reflexo de estiramento (Miscio et al., 2004). Diversas modalidades terapêuticas são utilizadas para se tentar minimizar a espasticidade. No entanto, estudos sugerem efeitos benéficos da estimulação somatossensorial, através da terapia vibratória (Beck et al., 2010).

Sabe-se que a vibração tem múltiplas influências fisiológicas, promovendo padrões normais de atividade motora pela modulação da excitabilidade dos motoneurônios. As vibrações aumentam o influxo aferente final do fuso muscular primário, permitindo a contração reflexa, o chamado reflexo tônico de vibração (Batista et al., 2007). Esse reflexo é mediado tanto por via espinhal segmentar quanto por via espinhal suprasegmentar, sendo sustentado por centros superiores (Hinselmann, 2006).

Recentemente foram desenvolvidos inúmeros aparelhos produtores de vibração, tais como halteres, plataformas vibratórias, almofadas, esteiras, dentre outros, e paralelamente à disseminação desses aparelhos, muitos estudos têm sido realizados para comprovar sua eficiência (Batista et al., 2007).

Em pacientes espásticos pós-acidente vascular encefálico, sabe-se que os estímulos vibracionais ativam o tecido conjuntivo, as fibras nervosas do tipo $\mathrm{Ia}, \mathrm{Ib}$, II, os receptores nervosos e regiões do sistema nervoso central, e se mostram potencialmente promissores, por levarem a um aumento da inibição pós-sináptica, atuando assim no músculo espástico, modulando o tônus, além de contribuir para a propriocepção através da retroalimentação dos receptores de estiramento, consequentemente, aumentando a funcionalidade (Gillies et al., 1969; Van Nes et al., 2004).
Grande parte dos sujeitos com lesão no SNC depara-se com problemas relacionados à espasticidade e fraqueza muscular, o qual interfere na sua função motora. Essas alterações dificultam o processo de reabilitação e limita, muitas vezes, padrões de movimentos funcionais.

Os benefícios do tratamento vibratório são previsíveis com base no conhecimento sobre os mecanismos neurofisiológicos. Sabe-se que as vibrações promovem padrões normais de atividade motora pela modulação da excitabilidade dos motoneurônios e da via córticoespinal. Desta maneira pode-se recomendar essa terapia para provocar efeitos de inibição da espasticidade e efeitos no controle motor em sujeitos com seqüela de AVE.

Assim, o objetivo deste estudo foi investigar os efeitos da estimulação vibratória, antes e após uma única sessão de aplicação desta técnica, através da variação do grau de amplitude de dorsiflexão do tornozelo através da goniometria e da ativação muscular através das leituras eletromiográficas do músculo tibial anterior e gastrocnêmio medial, do membro inferior do hemicorpo acometido, de sujeitos pós-AVE. O artigo se justifica não somente pela produção da ciência, mas também pela aplicação de recursos tecnológicos provenientes de diversas áreas, como a engenharia.

\section{Materiais e Métodos}

\section{Amostra}

Para compor a amostra foram selecionados vinte e sete sujeitos adultos, de ambos os sexos, apresentando seqüelas clínicas de AVE, entre o período de outubro e novembro de 2010, seguindo os seguintes critérios de inclusão: diagnóstico de AVE, comprovados por tomografia computadorizada ou ressonância magnética; ter tempo mínimo de doze meses de seqüela ou fase espástica estabelecida; apresentar espasticidade, classificada de 1 a 2 na Escala de Ashworth modificada; não apresentar doenças degenerativas e alterações ortopédicas na articulação do tornozelo; Os critérios de exclusão foram: Presença de alterações cárdio-respiratórias; Presença de disfasia ou afasia de Wernicke; fazer uso de medicamentos para promover relaxamento muscular; ter realizado treinamento vibratório anteriormente.

Após receber informações verbais e escritas, os sujeitos assinaram o termo de consentimento livre e esclarecido para participar do estudo. O projeto foi aprovado pelo Comitê de Ética em Pesquisa da Universidade do Vale do Paraíba-UNIVAP, sob o protocolo H39/CEP2010. 


\section{Intervenção}

Inicialmente foram coletados os dados de identificação pessoal dos voluntários. Em seguida, foram submetidos à avaliação clínica, para estabelecer sua inclusão no estudo, ou não.

Após serem admitidos na pesquisa, os pacientes foram solicitados a sentar em uma cadeira, de maneira relaxada. A partir daí realizou-se a goniometria na articulação do tornozelo para mensurar a amplitude de movimento articular, através do goniômetro da marca Carci, fabricado em plástico transparente com duas réguas de $20 \mathrm{~cm}$ e transferidor de $0^{\circ}$ $360^{\circ}$. O alinhamento goniométrico foi realizado e os sujeitos foram orientados a dorsofletir o pé ativamente, partindo de uma angulação de $0^{\circ}$ graus até o limite de angulação possível de ser realizada pelo sujeito. Os valores da amplitude foram coletados e registrados em tabela Excel. Logo após, o registro eletromiográfico superficial simultâneo do músculo tibial anterior e gastrocnêmio medial do membro inferior do hemicorpo comprometido foram coletados, por um tempo de 20 segundos, durante o qual o sujeito realizava movimento de dorsiflexão ativo-livre. Para captação do sinal, os sujeitos ficavam sentados, com os pés apoiados na almofada vibratória. Um par de eletrodos foi posicionado no ventre muscular do tibial anterior a $1 / 3$ da linha entre a cabeça da fíbula e o maléolo medial; o outro par na região mais proeminente do músculo gastrocnêmio medial e o eletrodo de referência foi fixado no epicôndilo lateral do cotovelo direito. Os eletrodos utilizados foram de $\mathrm{Ag} / \mathrm{AgCl}$ da marca Global Tec.

Dentre os parâmetros eletromiográficos, a raiz quadrada do valor médio quadrático (RMS-do inglês Root Mean Square) foi escolhido para inferir sobre os comandos fisiológicos do $\mathrm{SNC}$ ao músculo e sobre a relação entre o nível de ativação muscular.

Em seguida, o sujeito foi submetido a 15 minutos ininterruptos de vibração através da almofada vibratória digital da marca Nissan - Indústria e Comércio de Aparelhos Fisioterapêuticos LTDA, com uma frequencia de $80 \mathrm{~Hz}$ e amplitude de $1,8 \mathrm{~mm}$. A intervenção foi aplicada com os sujeitos na posição sentada em uma cadeira de forma relaxada, com os membros inferiores apoiados com base livre, pés descalços, joelhos flexionados e tornozelos em um ângulo de $90^{\circ}$ sobre a almofada vibratória. Ao término dos 15 minutos, a almofada foi desligada, coletouse de imediato 20 segundos de EMG, em seguida esperou-se 5 minutos e coletou-se mais 20 segundos e em seguida esperou-se mais 5 minutos e coletouse novamente 20 segundos de EMG e para finalizar realizou-se a goniometria. Para a coleta do sinal eletromiográfico foi utilizado o equipamento da marca
EMG System do Brasil LTDA, modelo EMG 410C, com taxa de amostragem de $2000 \mathrm{~Hz}$. Durante as coletas de eletromiografia e amplitude articular, o sujeito era orientado realizar o movimento de dorsiflexão ativolivre sendo que algumas precauções foram mantidas como a prevenção da movimentação das articulações do quadril e joelho, inversão e eversão do pé; prevenção do estiramento do músculo gastrocnêmio, através da manutenção de flexão do joelho.

As coletas aconteceram uma única vez para cada sujeito e por intermédio de um único avaliador.

Existem inúmeras técnicas descritas na literatura para processar o sinal do EMG bruto, visto que, este tem pouco valor para fazer uma correlação direta com o grau de atividade muscular (De Luca, 2002).

O processamento off-line do sinal eletromiográfico foi realizado por intermédio do software DelSys EMGworks analysis, constou-se inicialmente do uso de um filtro Band Stop, na faixa de $4^{\mathrm{a}}$ ordem Butterworth, e em seguida um filtro Band Pass, na faixa de $4^{\mathrm{a}}$ ordem Butterworth.

Considerando o tempo de 20 segundos para cada coleta, a análise dos dados foi realizada selecionando um trecho de 10 segundos de cada uma das aquisições. Considerando que o uso de filtros geralmente distorce o início e final do sinal, excluíram-se os 5 segundos iniciais e 5 segundos finais da coleta.

As características e amplitude e frequencia do sinal EMG têm se mostrado sensíveis a fatores intrínsecos, por exemplos: tipos de fibras, profundidade dos músculos; e extrínsecos como localização e tipos de eletrodos (De Luca, 2002). Portanto, a amplitude do sinal não pode ser analisada diretamente. Para se analisar e comparar sinais eletromiográficos de diferentes sujeitos e músculos faz-se necessário a utilização de técnicas de normalização.

No presente estudo os valores do RMS da EMG foram normalizados dividindo para cada sujeito os valores do RMS da EMG de cada fase, pelo valor do RMS na fase inicial antes da vibração. Esta forma de normalização permite comparar o valor do sinal da EMG nas diversas fases em relação ao valor inicial de cada músculo e também comparar a variação relativa dos músculos em estudo, entre si.

\section{Análise estatística}

A análise descritiva dos dados foi realizada por meio das medidas de tendência central e desvio padrão, valores máximo e mínimo. Os dados apresentaram uma distribuição normal, comprovados pelo teste de normalidade de D’Agostino. A análise de variância (ANOVA-ANalysis Of VAriance) para amostras relacionadas foi utilizada para comparação dos resultados antes e após a utilização do recurso. 
Identificada as diferenças entre a atividade eletromiográfica antes e após o recurso, era usado o teste post hoc de Tukey para identificar quais médias eram estatisticamente diferentes. O Teste $t$-Student pareado foi aplicado para comparar os resultados da goniometria antes e depois da vibração. Atribuiu-se para os testes estatísticos o nível de significância de 5\%. Os testes estatísticos foram realizados utilizando o software BioEstat 5.0.

\section{Resultados}

\section{Caracterização da amostra}

Participaram do estudo 27 pacientes, média de idade de 64,4 $\pm 4,24$ (46-75 anos) e com tempo de AVE de $33,7 \pm 12,00$ (12-60 meses), sendo 25 homens e 2 mulheres, quanto ao tipo de AVE, 17 foram de etiologia isquêmica e 10 hemorrágicos. Em relação ao hemicorpo comprometido, 17 apresentavam hemiparesia direita e 10 hemiparesia esquerda.

\section{Goniometria}

A Figura 1 apresenta os resultados da amplitude articular no pré e pós à aplicação da vibração para o movimento de dorsiflexão do tornozelo. Houve um aumento estatisticamente significativo após a terapia vibratória $(\mathrm{p}<0,0001)$.

\section{Atividade eletromiográfica}

A Figura 2 apresenta os resultados do RMS após a aplicação da vibração, normalizado pelo valor do pré-vibração no músculo tibial anterior. Houve um aumento estatisticamente significativo após a terapia vibratória $(\mathrm{p}=0,005)$.

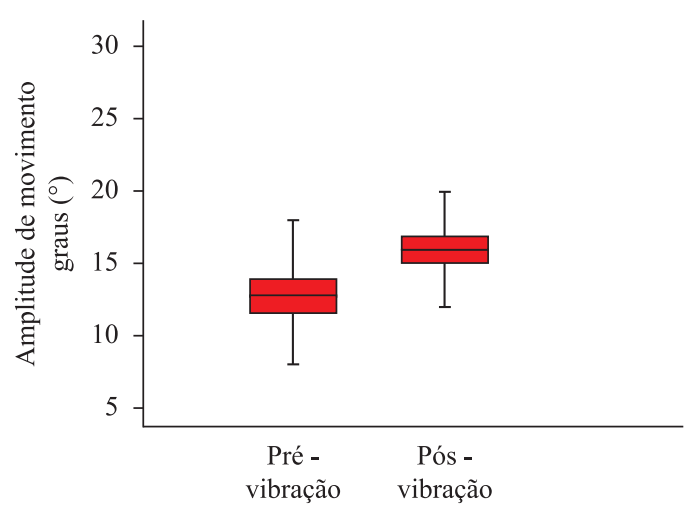

Figura 1. Valores da amplitude articular (em graus), do movimento de dorsiflexão, antes e após a vibração. Valores apresentados em média, \pm dois desvios padrão, valores máximo e mínimo $(\mathrm{p}<0,0001)$. Figure 1. Range of motion (in degree) of the dorsiflexion motion, before and after vibration. Values are represented in mean, \pm two standard deviation, maximum and minimum values $(p<0.0001)$.
Quando comparado o pós-imediato e pós 5 e 10 minutos do término da vibração, notou-se um aumento significativo $(\mathrm{p}<0,01)$, porém, quando comparado o valor do pós-5 minutos e pós-10 minutos da vibração não houve diferença significativa.

A Figura 3 apresenta os resultados do RMS normalizado no pré e pós a aplicação da vibração no músculo gastrocnêmio medial. Houve um aumento estatisticamente significativo após a terapia vibratória $(\mathrm{p}=0,005)$.

Os valores RMS normalizado do EMG do músculo gastrocnêmio medial, não mostraram diferenças significativas pós-imediato da aplicação da vibração, porém mostraram diferenças significativas, após 5 minutos do término da vibração $(\mathrm{p}<0,05)$ e após 10 minutos do término do recurso $(\mathrm{p}<0,01)$.

\section{Discussão}

O músculo esquelético é um tecido especializado que modifica sua capacidade funcional em resposta a diferentes estímulos. $\mathrm{O}$ corpo humano responde à vibração de forma complexa, pois esta impõe uma atividade de hipergravidade em função de altas acelerações. A ação mecânica da vibração é realizada para produzir rápidas e curtas mudanças no comprimento do complexo músculo-tendíneo. Essa perturbação é detectada por receptores sensoriais, que modulam a rigidez muscular através de uma atividade reflexa tentando amortecer as ondas vibratórias (Cardinale e Bosco, 2003).

$\mathrm{O}$ aumento observado no grau de amplitude de movimento de dorsiflexão e do aumento do RMS do sinal eletromiográfico do músculo tibial anterior, estatisticamente significativo, após a vibração, foi

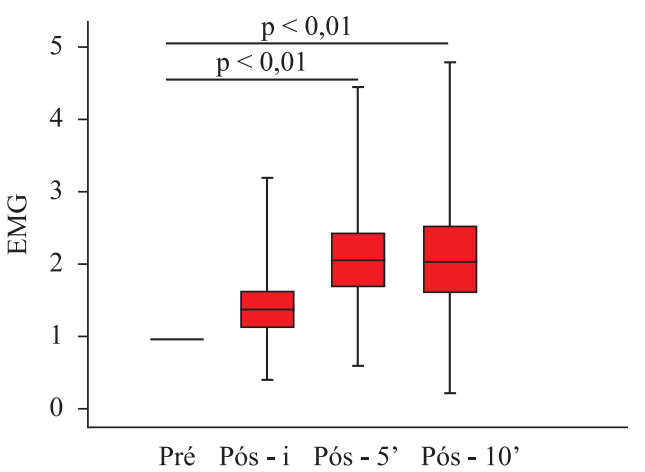

Figura 2. Valores RMS normalizado da EMG do músculo tibial anterior, pré-vibração, pós-vibração imediato, pós-vibração 5 minutos, pós-vibração 10 minutos. Valores apresentados em média, \pm dois desvios padrão, valores máximo e mínimo $(\mathrm{p}=0,005)$.

Figure 2. Normalised tibialis anterior muscle EMG RMS, before vibration, immediately after vibration, 5 minutes after vibration, and 10 minutes after vibration. Values are represented in mean, \pm two standard deviation, maximum and minimum values $(p=0.005)$. 


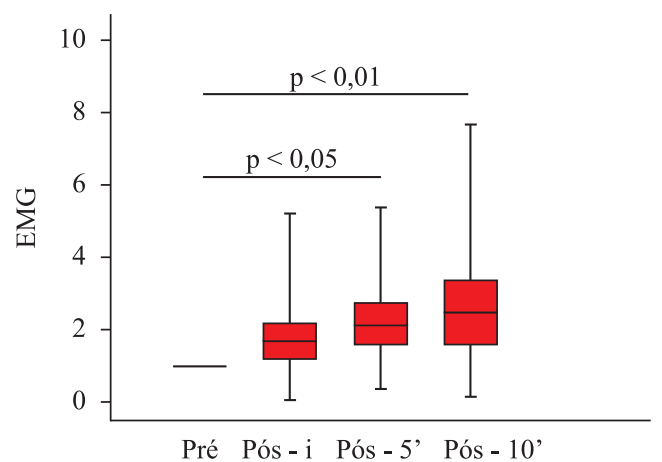

Figura 3. Valores RMS normalizados da EMG do músculo gastrocnêmio medial, pré-vibração, pós-vibração imediato, pósvibração 5 minutos, pós-vibração 10 minutos. Valores apresentados em média, \pm dois desvios padrões, valores máximo e mínimo $(\mathrm{p}=0,005)$. Figure 3. Normalised gastrocnemius muscle EMG RMS, pre-vibration, immediate post-vibration, five-minute post-vibration, ten-minute postvibration. Values are presented in mean, \pm two standard deviation, maximum and minimum values $(p=0.005)$.

indicativo de melhora da função do membro, fato que deve estar correlacionado ao recurso utilizado, pois este visou incrementar a mobilidade por provocar, mesmo que temporariamente, mudanças na função das unidades motoras dos músculos agonistas, modulação da espasticidade do músculo antagonista e controle motor voluntário dos sujeitos. Os dados obtidos são compatíveis com as observações de pesquisadores como Cardinale e Bosco (2003); Ortolan et al. (2005); Cordo et al. (2009).

Esperava-se nesse estudo, baseado nos indícios neurofisiológicos, que houvesse aumento do sinal eletromiográfico do músculo tibial anterior (agonista) concomitante à redução para o músculo gastrocnêmio medial (antagonista) durante o movimento de dorsiflexão. No entanto houve um aumento do RMS (pós-5 min e 10 min do término da vibração) para o músculo gastrocnêmio medial, estatisticamente significativo. Isso não é indicativo do aumento da cocontração e nem do aumento da espasticidade desse músculo. De acordo com o protocolo experimental desse estudo o sinal eletromiográfico foi captado durante a realização da dorsiflexão e durante a ação de retorno do pé a posição neutra. As ações do gastrocnêmio são de flexão plantar e flexão de joelho (Shumway-Cook e Woollacott, 2003). Durante a coleta, o sujeito encontrava-se em flexão de joelho e durante o retorno a posição neutra do movimento de dorsiflexão, esse músculo é esperado por demonstrar maior ativação que os demais flexores plantares (Abdullah et al., 2008), principalmente quando o músculo tibial anterior apresenta déficit de contração excêntrica (Shumway-Cook e Woollacott, 2003). Além disso, o aumento da ativação muscular do gastrocnêmio medial pode estar relacionada à resposta excitatória, ocasionada por uma ilusão de movimento provocada pela vibração, chamada de reflexo tônico de vibração inverso, que ativa os músculos antagonistas. Concordando com o estudo de Calvin-Figuière et al., (1999).

O aumento do RMS do músculo tibial anterior após a vibração é justificado pelo aumento da eficiência neuromuscular. A vibração induz alterações temporárias na excitabilidade de estruturas neurais centrais e periféricas (Bosco et al., 2000; Cardinale e Bosco, 2003; Cunnington et al., 2002; Naito et al., 2005; Rittweger et al.,2003), o que supostamente, facilitaria a ativação de unidades motoras nos minutos subsequentes, e explicaria as melhoras no desempenho com treinamento de vibração (Armstrong et al., 2008; Cardinale e Bosco, 2003; Mileva et al., 2009; Rittweger et al., 2003).

$\mathrm{Na}$ reabilitação, a aplicação de estímulo proprioceptivo promove padrões de atividade motora pela modulação da excitabilidade dos motoneurônios projetando eferências para os músculos. A organização cortical pode ser remodelada secundária à alteração de entradas aferentes por alteração da representação cortical associada à região estimulada (Lorraine $\mathrm{e}$ Brenda, 2005; Smith e Brouwer, 2005).

Vale considerar que as principais limitações desse estudo foram não considerar o numero de vezes que o sujeito poderia realizar o movimento de dorsiflexão, dentro do tempo estimado. Além disso, não se incluiu medidas de avaliação funcional, o que teria permitido interpretar os resultados de acordo com seu significado clínico.

\section{Conclusões}

Diante dos resultados, de acordo com o protocolo experimental utilizado, foi demonstrado que a vibração proporcionou aumento do grau de amplitude de movimento de dorsiflexão do tornozelo, aumento da atividade eletromiográfica para o músculo tibial anterior e gastrocnêmio medial após a aplicação da estimulação vibratória. Outros estudos são necessários para o conhecimento dos efeitos da estimulação vibratória diante dos parâmetros físicos.

\section{Referências}

Abdullah NA, Abuosman NA, Abdulrahim RB. The effects of ankle-foot orthosis (AFO) on electromyography muscles activity. In: The 3th International Symposium on Biomedical Engineering - ISBME: Proceedings of the International Symposium on Biomedical Engineering; 2008 nov. IEEE; 2008.p.101-4.

Armstrong WJ, Nestle HN, Grinnell DC, Cole LD, Van Gilder EL, Warren GS, Capizzi EA. The acute effect of whole-body 
vibration on the Hoffmann reflex. Journal of Strength and Conditioning Research. 2008; 22(2):471-6. PMid:18550962. http://dx.doi.org/10.1519/JSC.0b013e3181660605

Batista MAB, Wallerstein LF, Dias RM, Silva RG, Ugrinowitsch C, Tricoli V. Efeito do Treinamento com plataformas vibratórias. Revista Brasileira Ciência e Movimento. 2007; 15(3):103-13.

Bear MF, Connors BW, Paradiso MA. Neurociências: Desvendando o sistema nervoso. 2th ed. Porto Alegre: Artmed; 2002.

Beck EK, Neto GNN, Nohama P. Estímulo vibracional na espasticidade - Uma perspectiva de tratamento. Revista de Neurociências. 2010; 18(4):523-30. http://dx.doi.org/10.4181/ RNC.2010.ip02.7

Bosco C, Lacovelli M, Tsarpela O, Cardinale M, Bonifazi M, Tihanyi J, Viru M, De Lorenzo A, Viru A. Hormonal to whole-body vibration in men. European Journal of applied physiology. 2000; 81(6):449-54. PMid:10774867. http://dx.doi.org/10.1007/s004210050067

Calvin-Figuière $\mathrm{S}$, Romaiguère $\mathrm{P}$, Gilhodes JC, Roll JP. Antagonists motor responses correlate with kinesthetic illusions induced by tendon vibration. Experimental Brain Research. 1999; 124:342-50. http://dx.doi.org/10.1007/ s002210050631

Cardinale M, Bosco C. The use of vibration as an exercise intervention. Exercise and Sport Sciences Reviews. 2003; 31(1):3-7. PMid:12562163. http://dx.doi. org/10.1097/00003677-200301000-00002

Cordo P, Lutsep H, Cordo L, Wright W, Cacciatore T, Skoss R. Assisted movement with enhanced sensation (AMES): Coupling motor and sensory to remediate motor deficits in chronic stroke patients. Neuro Rehabilitation and Neural Repair. 2009; 23(1):67-77. PMid:18645190. http://dx.doi. org/10.1177/1545968308317437

Corrêa FI, Soares F, Andrade DV, Gondo RM, Peres JA, Fernandes AO, Corrêa JC. Atividade muscular durante a marcha após acidente vascular encefálico. Arquivo de Neuropsiquiatria. 2005; 63(3b):847-51. PMid:16258668.

Cunnington R, Windischberger C, Deecke L, Moser E. The preparation and execution of self-initiated and externally-triggered movement: a study of event-related fMRI. Neuroimage. 2002; 15(2):373-85. PMid:11798272. http://dx.doi.org/10.1006/nimg.2001.0976

De Luca CJ. Surface Electromyography: Detection and Recording. Delsys Incorporated [Internet]. 2002 [Cited 2011 April 04]; [About 10 p.]. Available from: http://www.delsys. com/Attachments_pdf/WP_SEMGintro.pdf

Gillies JD, Lance JW, Neilson PD, Tassinari CA. Presynaptic inhibition of the monosynaptic reflex by vibration. Journal of Physiology. 1969; 205:329-39. PMid:5357239. PMCid:1348605.
Hinselmann F. Verificação da interferência da vibração associada à terapia neurofuncional na melhora do controle postural na criança portadora de paralisia cerebral do tipo hemiparética espástica: um estudo comparativo [monografia]. Cascavel: Faculdade Assis Gulgacz; 2006. 76 p.

Lorraine S, Brenda B. Effectiveness of muscle vibration in modulating corticospinal excitability. Journal of Rehabilitation Research \& Development. 2005; 42(6):787-94. http://dx.doi. org/10.1682/JRRD.2005.02.0041

Mileva KN, Bowtell JL, Kossev AR. Effects of lowfrequency whole-body vibration on motor-evoked potentials in healthy men. Experimental Physiology. 2009; 94(1):10316. PMid: 18658234 . http://dx.doi.org/10.1113/ expphysiol.2008.042689

Miscio G, Del Conte C, Pianca D, Colombo R, Panizza M, Shieppati M, Pisano F. Botulinum toxyn is post-stroke patients: stiffness modifications and clinical implications. Journal of Neurology. 2004; 251(2):189-96. PMid:14991354. http://dx.doi.org/10.1007/s00415-004-0297-3

Naito E, Boetell JL, Kossev AR. Effects of low-frequency whole-body vibration on motor-evoked potentials in healthy men. Experimental Physiology. 2005; 94(1):103-16.

Ortolan RL, Reis GS, Magro LS, Koeke PU, Parizotto NA, Cliquet JRA. Tratamento de terapia vibratória em pacientes com espasticidade. Fisioterapia em Movimento. 2005; 18(1):67-74.

O`Sullivan SB, Schmitz TJ. Fisioterapia: avaliação e tratamento. 5th ed. São Paulo: Manole; 2010.

Rittweger J, Mutschelknauss M, Felsenberg D. Acute changes in neuromuscular excitability after exhaustive whole body vibration exercise as compared to exhaustion by squatting exercise. Clinical Physiology and Functional Imaging. 2003; 23(2)81-6. PMid:12641601. http://dx.doi. org/10.1046/j.1475-097X.2003.00473.x

Shumway-Cook A, Woollacott MH. Controle motor: teoria e aplicações práticas. 2th ed. São Paulo: Manole; 2003.

Smith L, Brouwer B. Effectiveness of muscle vibration in the modulation of excitability corticospinal. Journal of Rehabilitation Research \& Development. 2005; 42(6):787-94. http://dx.doi.org/10.1682/JRRD.2005.02.0041

Soares AV. A combinação da facilitação neuromuscular proprioceptiva com o biofeedback eletromiográfico na recuperação do pé caído e na marcha de paciente com acidente vascular cerebral. Fisioterapia em Movimento. 2003; 16(2):61-72.

Van Nes IJW, Geurts ACH, Hendricks HT, Duysens J. Short-term effects of whole-body vibration on postural control in unilateral chronic stroke patients: preliminary evidence. American Journal of Physical Medicine and Rehabilitation. 2004; 83(11):867-73. http://dx.doi. org/10.1097/01.PHM.0000140801.23135.09

\section{Autores

